\title{
Retos de la COVID-19
}
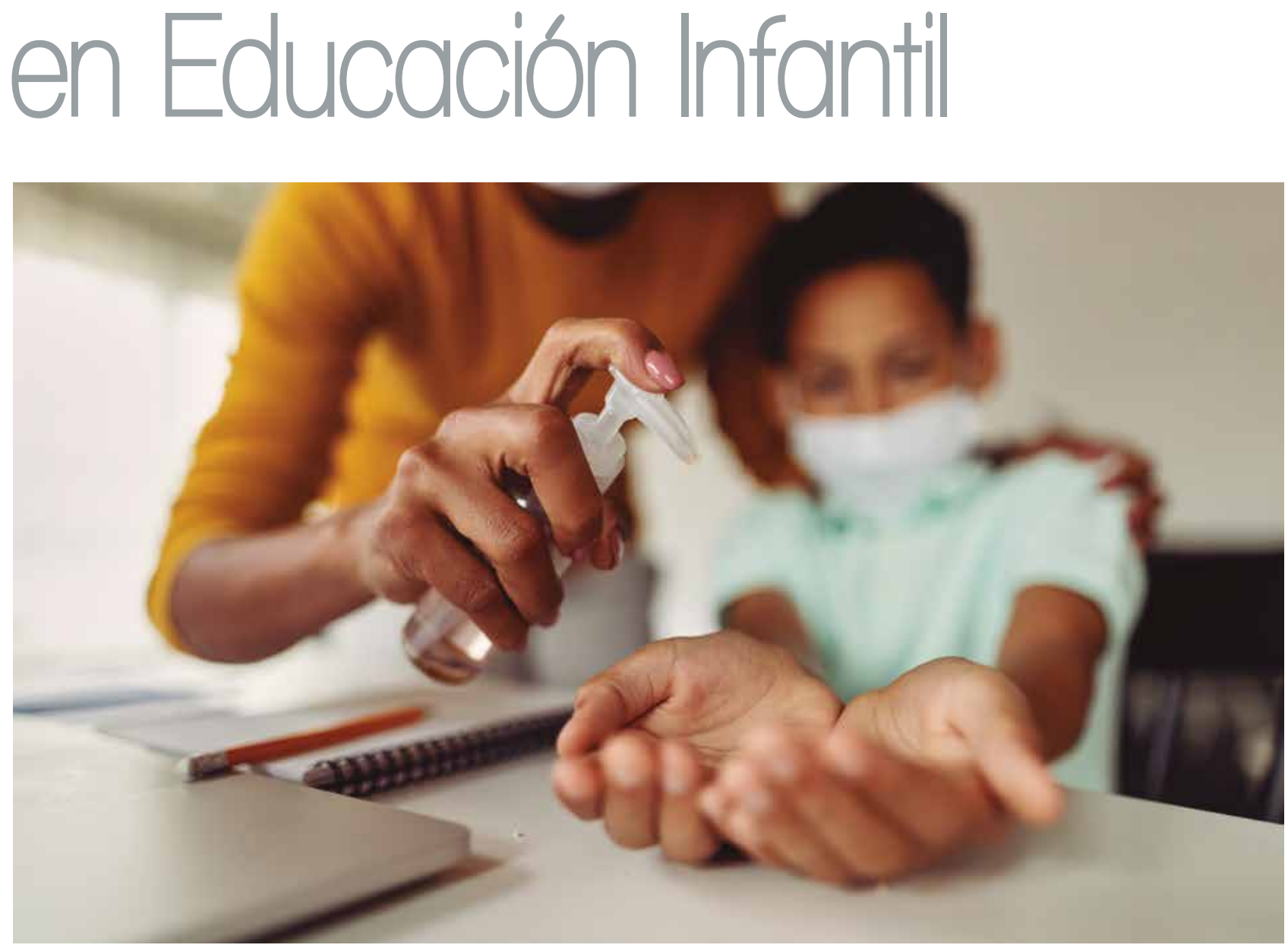

La pandemia y la llegada de los

confinamientos nos ha afectado a cada uno de una manera muy distinta, pero para los alumnos de Infantil ha sido un reto poder continuar con su educación. Los profesores,

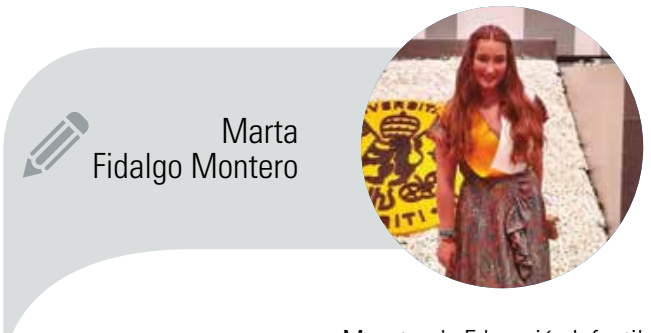
todo el personal de los colegios y las familias han tenido que ir superando retos, pero al final han conseguido reinventar la educación y crear un espacio donde cada uno de los alumnos se desarrolle al máximo posible. 
Quién me iba a decir a mí, que mis últimas prácticas de la carrera iban a ser en mitad de una pandemia mundial, donde las mascarillas y los geles hidroalcohólicos serían los nuevos aliados de los docentes.

El comienzo del curso estuvo marcado por la incertidumbre, por el miedo a volver a salir a la calle, pero, sobre todo, por las ganas de recuperar aquello que habíamos perdido el 13 de marzo de 2020.

Recuerdo hablar con mis amigas y comentar si este año sería posible hacer prácticas en algún colegio, si alguien estaría dispuesto a dejar entrar en un aula de Infantil a una auténtica desconocida. Para nuestra sorpresa, y gracias a la labor de la universidad y de los colegios, el 3 de noviembre estábamos empezando nuestras últimas prácticas antes de convertirnos en maestras.

En mi caso, comencé en el Colegio Gaudem, situado en el distrito de Barajas en un aula de 4-5 años, las Estrellas rojas. Todavía me sigo acordando de la sensación que tuve el primer día al llegar, nada de saludos, distancia con los alumnos, nuevas presentaciones con mascarilla que te obligaban a recrear las caras de las personas..., pero me invadió un sentimiento de felicidad; felicidad de poder volver a estar en un aula, aunque esta vez en otras circunstancias.

Los alumnos del colegio demostraron gratamente el cumplimiento de todas las

\section{Retos del docente en educación virtual}

\section{Conocimiento de las TIC}

\section{Motivación y atención del alumnado}

Coordinación entre los profesionales

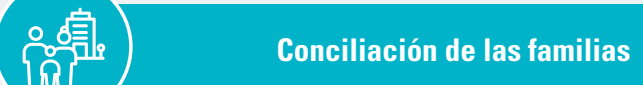

normas y protocolos que estaban impuestos. Cada clase era un espacio burbuja y sabían que tenían que estar con los amigos de su grupo sin juntarse con los demás. Esto puede resultar algo fácil, pero para un niño de Infantil acostumbrado a corretear por el patio, jugar con todos los niños sin importar de qué clase sean... es una tarea compleja.

Hablando con profesores de distintos colegios y alumnos en prácticas, coincidían en que la actuación de los alumnos frente a la COVID-19 estaba siendo impecable y que cada uno de ellos resaltaba su alegría de poder estar dando clases presenciales en lugar de online mediante un ordenador, tablet o móvil. Tanto es así, que la Comunidad de Madrid les ha otorgado la Gran Cruz de la Orden del Dos de Mayo por su actuación, trabajo y ejemplo.

En la etapa de Educación Infantil, ha sido complejo poder Ilevar a cabo las clases de una manera online. El mayor impedimento a la hora de plantear la enseñanza online es que tanto la etapa de Educación Infantil como la etapa de Educación obligatoria y Bachillerato no están pensadas para llevarlas a cabo con esta metodología, siempre se han mantenido en un formato presencial con algunas prácticas virtuales, como puede ser la metodología de flipped classroom. Otras dificultades que se pueden encontrar a la hora de adaptar el formato presencial al virtual es la corta edad de los alumnos o el currículo oficial en el que solo se contempla un acercamiento a las nuevas tecnologías y no el uso de ellas.

La necesidad de no paralizar a los alumnos debido a la pandemia o los confinamientos selectivos de clase han dado lugar a muchas reuniones, propuestas, alternativas... en las que administradores, equipos directivos, coordinadores de ciclo y de nivel y, por supuesto, los profesores han puesto todo su empeño para que los alumnos tuvieran una rutina parecida a la que realizarían en el colegio.

Los profesores a la hora de llevar a cabo las clases en línea se han encontrado con algunas dificultades, de las que en mi opinión y experiencia destaco cuatro. 


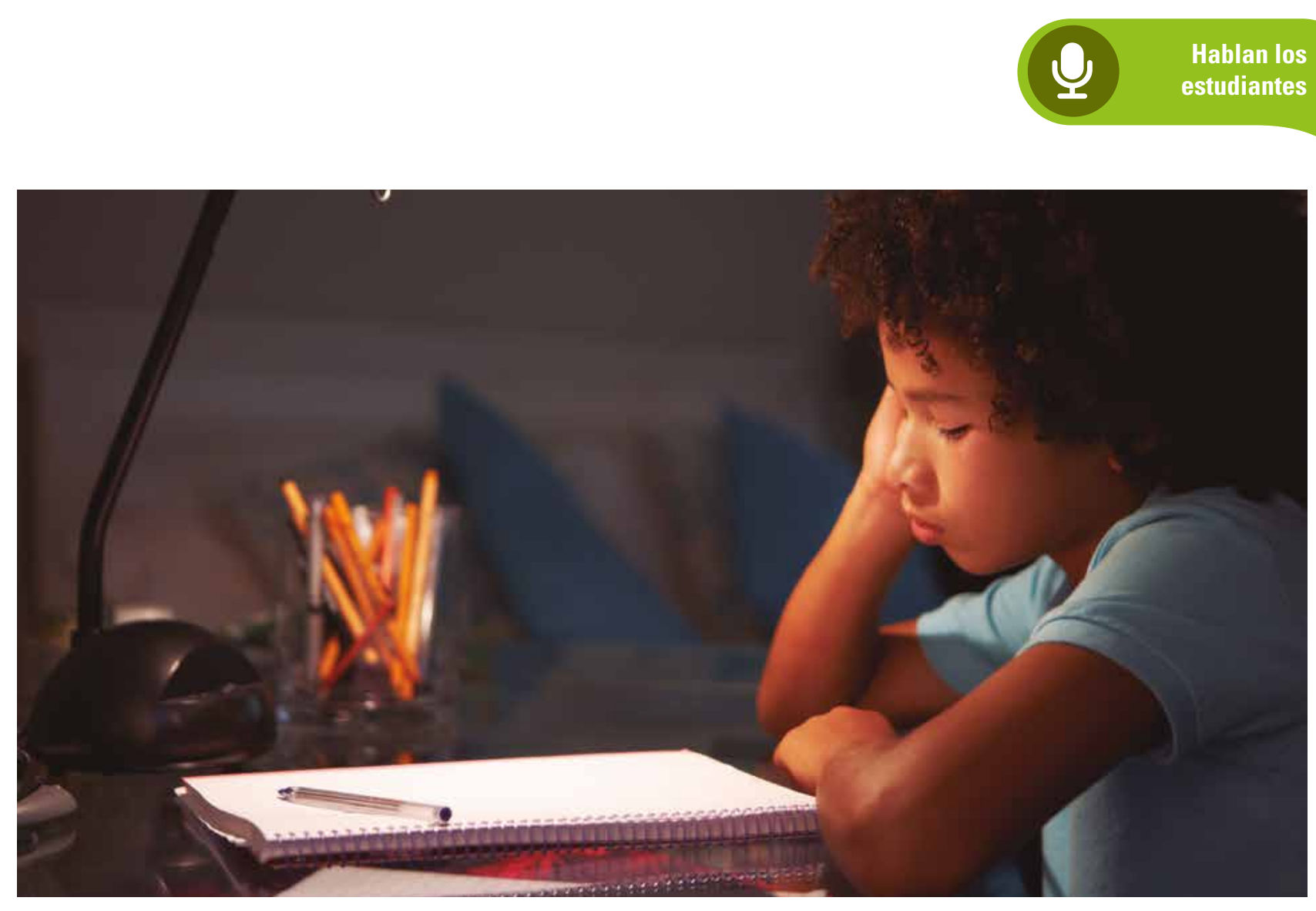

La primera de ellas es el aprendizaje y actualización de los conocimientos de tecnología, ya que muchos de los maestros nunca habían tenido que dar una clase mediante una pantalla, o grabarse explicaciones para que sus alumnos hagan las actividades programadas. A esto, se le suma la necesidad de conocer nuevas aplicaciones para crear contenido ameno, lúdico y dinámico para alumnos pequeños, donde necesiten la menor ayuda posible y se fomente su autonomía. Algunas de las aplicaciones que más se han usado a lo largo de los meses de confinamiento y del cierre de las aulas una vez iniciado el curso escolar han sido: Google Classroom, Smile and Learn o Liveworksheets.

La segunda dificultad ha sido la atención y la motivación de los alumnos. El modelo online no debe suponer una pérdida de la calidad en el proceso de enseñanzaaprendizaje por lo que es necesario que nuestro alumnado tenga ganas de aprender y tenga una predisposición hacia ello. Como docentes tenemos el deber de buscar las herramientas necesarias para que la metodología y los recursos que creemos estén personalizados y pensados para el tipo de alumnado que tenemos. La atención debe ser una de nuestras prioridades, no podemos olvidarnos de trasmitir aqueIlo que queremos que les llegue a nuestros alumnos, de expresar con el cuerpo y, sobre todo, debemos intentar no perder la

\section{Cuando los alumnos acuden al colegio están en las mismas condiciones de aprender, pero con la pandemia se ha incrementado la necesidad de que haya lecnología en todos los hogares para no perder el derecho a la educación}

construcción colectiva de nuevos aprendizajes, aunque ellos solo nos vean la cara por estar detrás de una cámara.

El tercero de los retos de los docentes es la coordinación con el equipo de profesionales, seguir manteniendo reuniones de nivel y de etapa para compartir experiencias, buscar soluciones a problemas que surgen durante las conexiones con los niños y coordinarse a la hora de seguir un plan conjunto para las clases online.

Por último, la cuarta dificultad sería la conciliación familiar. La mayoría de los alumnos de Infantil no son autónomos como para encender el ordenador, buscar en Google los documentos que les ha enviado su profesor o conectarse a una videollamada. La actuación de los padres en este caso es imprescindible y vuelve a ponerse en alza la importancia de la compenetración y comunicación de la familia y la escuela. Los profesores deberán tener 


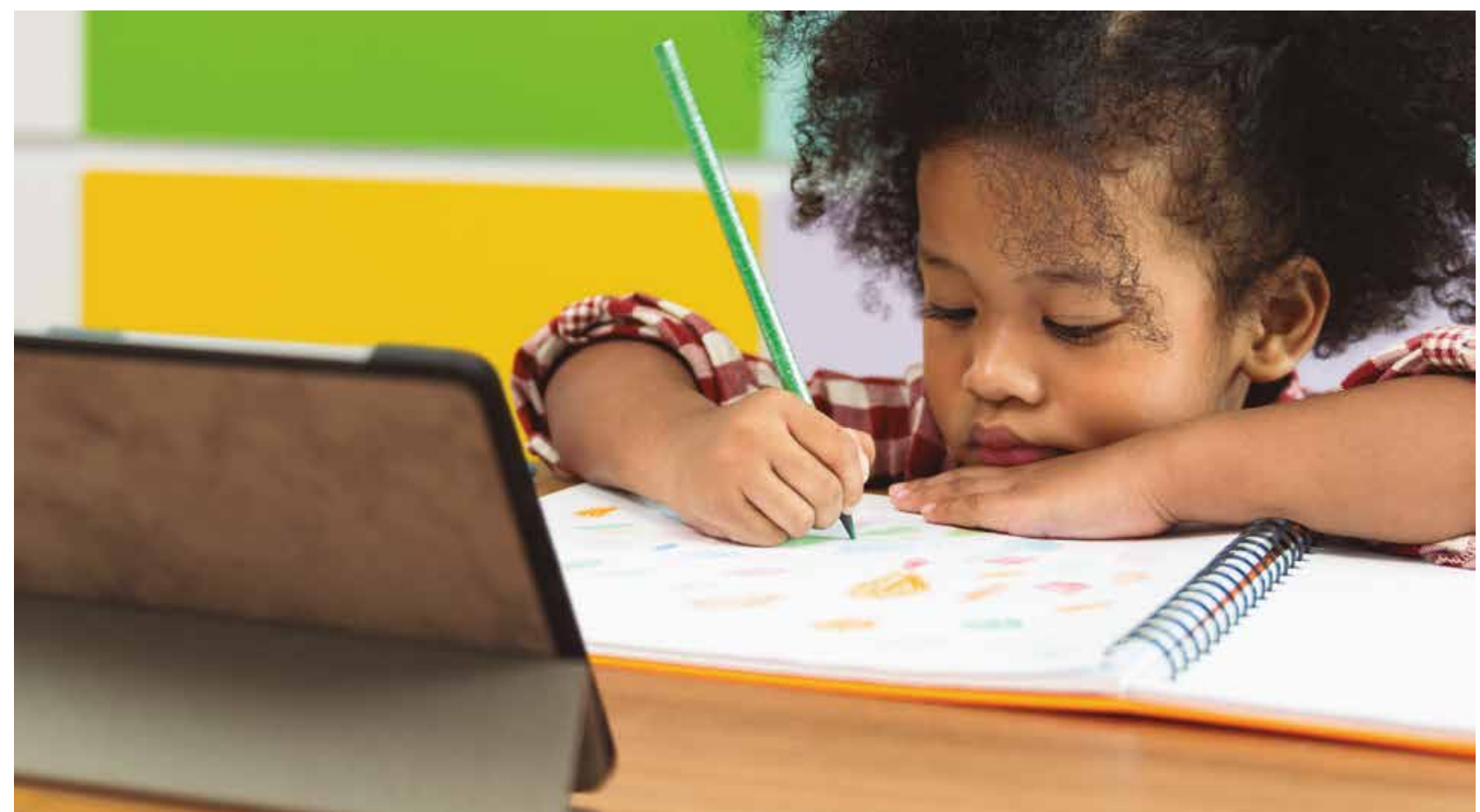

\section{Brecha digital}

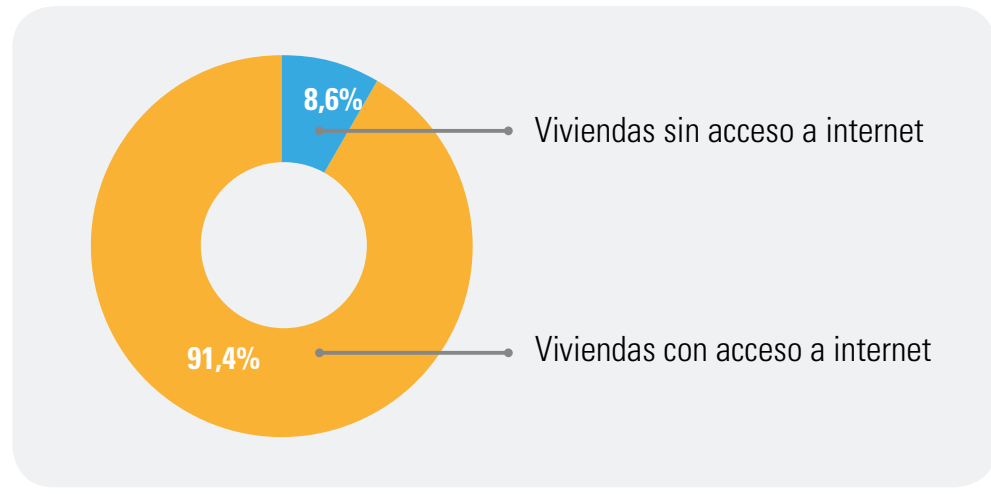

Fuente: elaboración propia medio para conectarse a internet frente al $8,6 \%$ que no disponen de ningún recurso para ello, esto supone que una de cada diez familias está desconectada y aislada del mundo.

Cuando los alumnos acuden al colegio están en las mismas condiciones de aprender, pero con la pandemia se ha incrementado la necesidad de que haya tecnología en todos los hogares para poder continuar con el ritmo de las clases y no perder el derecho a la educación.

Antes de concluir con este artículo, debemos comentar aquellos retos que han supuesto para el alumnado de Educación Infantil seguir las clases desde una pantalla, así como las consecuencias que ha tenido para ellos y su aprendizaje.

La escuela para los alumnos, sobre en cuenta que puede haber padres teletrabajando a los que les resulta imposible ponerse con su hijo a hacer los ejercicios, a practicar la lectura o a crear alguna manualidad. Para ello es importante que se propongan alternativas como dejar videotutoriales explicando cómo hacerlo para que los niños vayan imitando a la vez que lo ven o proponer actividades en las que no necesiten de ayuda adulta.

Uno de los mayores retos a los que se ha tenido que enfrentar el profesorado ha sido a la brecha digital que ha surgido en nuestro país debido a la situación de la COVID-19. Según un estudio el 91,4\% de la población española tiene algún tipo de todo para los pequeños, supone un espacio de desarrollo emocional, personal, pero lo más importante social. En los colegios es donde nuestros alumnos pueden aumentar la conciencia social, el pensamiento de colectividad y de conjunto, desarrollando las aptitudes y actitudes necesarias para la vida en sociedad.

Con la llegada de la COVID-19 muchos niños han presentado un sentimiento de vacío, de soledad, ya que no podían desarrollar esas habilidades sociales tan necesarias para su edad. Muchos pueden tener ese sentimiento por no ver a sus abuelos, primos e incluso a sus amigos del colegio. 
Tabla de tiempos de uso de la tablet según la edad

\begin{tabular}{|c|c|c|}
\hline Edad & Tiempo & Consecuencias \\
\hline Menos de 2 años & Nunca & Falta de capacidad de aprendizaje \\
\hline Menos 2-5 años & 30 minutos & Falta de control de sentimientos \\
\hline Menos 5-12 años & 60 minutos & Sedentarismo y obesidad \\
\hline Más de 12 años & 2 horas & Afecta al rendimiento escolar y las habilidades sociales \\
\hline
\end{tabular}

Fuente: FERNÁNDEZ, P. (2018). Tabla de tiempo de uso de la tablet o móvil en niños según su edad. Guiainfantil.com. https:I/www. guiainfantil. com/blog/educacion/nuevas-tecnologias/el-tiempo-de-uso-de-la-tablet-o-movil-en-ninos-segun-su-edad-resumido-en-una-tablal

Los docentes en este caso pueden aprovechar esos espacios de encuentro para que cada uno les cuente a sus compañeros algo especial que haya hecho ese día o les dedique unas palabras a sus amigos para conseguir esa sensación de pertenencia a un grupo.

Uno de las peores consecuencias derivadas de esta situación y que estamos empezando a percibir es la adicción a las pantallas. Durante mi periodo de prácticas he visto a niños obsesionados con los iPads dejando de lado los juguetes tradicionales como pueden ser la cocinita, los coches, los bebés o las construcciones. Este es un reto muy difícil para los maestros y las familias ya que necesitamos de las tecnologías para comunicarnos con ellos, pero hay que encontrar un término medio para que no generen dependencia hacia los dispositivos electrónicos.

En mi experiencia durante este año diferente, he aprendido que es necesario hacer sentirse importante a cada uno de los alumnos que tienes detrás de los ordenadores, porque para ellos es un momento muy relevante, ver a su profesora, a sus amigos...

\section{HEMOS HABLADO DE}

\section{Educación Infantil; nuevas tecnologías; COVID-19; motivación; retos.}

Este artículo fue solicitado por PADRES Y MAESTROS en marzo de 2021, revisado y aceptado en mayo de 2021.
Considero que podría ser una buena práctica dejar un tiempo pequeño para que cada uno resalte algo que quiera, que se sienta importante durante ese momento del día y se olvide de la situación que está viviendo. Este momento puede crear un clima óptimo de aprendizaje donde el alumno está motivado, concentrado y puedes realizar actividades con ellos.

En conclusión, durante estos meses de pandemia nos hemos visto sobrellevados por una situación para la que no estábamos preparados, pero que ha conseguido que la educación se reinvente y cree nuevas formas para llegar a los más pequeños •

\section{(D) DARA SABER MÂS}

Saldaña Montero, J. (2020). Educación Infantil y enseñanza online durante el confinamiento: experiencias y buenas prácticas. Etic@net, 20(2),336-348. http://dx8.doi. org/10.30827/eticanet.v20i2.16214

Cifuentes-Faura, J. (2020). Consecuencias en los Niños del Cierre de Escuelas por Covid-19: El Papel del Gobierno, Profesores y Padres. Revista Internacional de Educación para la Justicia Social, 9(3). https://revistas.uam.es/riejs/article/ view/12216

Cabrera L. (2020). Efectos del coronavirus en el sistema de enseñanza: aumenta la desigualdad de oportunidades educativas en España. Revista de Sociología de la Educación (RASE) 2020, 13(2), 114-139. http:// dx. doi.org/10.7203/RASE.13.2.17125 